\title{
Current Use of Intracoronary Imaging in Interventional Practice - Results of a European Association of Percutaneous Cardiovascular Interventions (EAPCI) and Japanese Association of Cardiovascular Interventions and Therapeutics (CVIT) Clinical Practice Survey -
}

\author{
Konstantinos C. Koskinas, MD; Masato Nakamura, MD; Lorenz Räber, MD, PhD; \\ Roisin Colleran, MD; Kazushige Kadota, MD; Davide Capodanno, MD, PhD; \\ William Wijns, MD, PhD; Takashi Akasaka, MD; Marco Valgimigli, MD, PhD; \\ Giulio Guagliumi, MD; Stephan Windecker, MD; Robert A. Byrne, MD, PhD
}

Background: This study evaluated the views of the cardiology community on the clinical use of coronary intravascular imaging (IVI).

Methods and Results: A web-based survey was distributed to 31,893 individuals, with 1,105 responses received (3.5\% response rate); 1,010 of 1,097 respondents $(92.1 \%)$ self-reported as interventional cardiologists, $754(68.7 \%)$ with $>10$ years experience. Overall, $96.1 \%$ had personal experience with IVI (95.5\% with intravascular ultrasound [IVUS], 69.8\% with optical coherence tomography [OCT], and $7.9 \%$ with near-infrared spectroscopy); $34.7 \%$ of respondents were from Europe and $52.0 \%$ were from Asia (45.4\% from Japan). The most commonly reported indications for IVI were optimization of stenting (88.5\%), procedural/strategy guidance (79.6\%), and guidance of left main interventions (77.0\%). Most respondents reported perceived equipoise regarding choice between IVUS and OCT for guidance of coronary intervention. High cost (65.9\%) and prolongation of the procedure (35.0\%) were the most commonly reported factors limiting use. IVI was used more frequently (>15\% of cases guided by IVI) in Japan than Europe (96.6\% vs. $10.4 \%$, respectively; $\mathrm{P}<0.001)$ and by operators with longer interventional experience.

Conclusions: In a sample of predominantly experienced interventional cardiologists, there was a high rate of personal experience with IVI in clinical practice. The most commonly identified indications for IVI were optimization of stenting, procedural/strategy guidance, and guidance of left main interventions. Variability in practice patterns is substantial according to geographic region and interventional experience.

Key Words: Coronary imaging; Intravascular ultrasound; Optical coherence tomography

I n past decades, coronary intravascular imaging (IVI) has evolved from a primary research tool to a relatively frequently used adjunctive diagnostic modality in clinical practice. ${ }^{1} \mathrm{~A}$ growing body of evidence supports a role for intravascular ultrasound (IVUS) and optical coherence tomography (OCT) in optimizing procedural results, ${ }^{2,3}$ and improving clinical outcomes of selected patients undergoing percutaneous coronary interventions (PCIs). ${ }^{4}$ In current
European clinical practice guidelines, IVUS imaging is recommended to optimize stent implantation in selected patients and to assess the severity and optimize treatment of left main lesions (Class IIa). ${ }^{5}$ In addition, both IVUS and OCT are recommended for the assessment of mechanisms of stent failure (Class IIa). ${ }^{5}$ IVI modalities also permit quantification of atheroma burden and characterization of coronary plaque composition in vivo. ${ }^{1}$ Lesions with

The article has been co-published with permission in EuroIntervention [DOI:10.4244/EIJY18M03_01] and Circulation Journal [DOI:10.1253/circj.CJ-17-1144]. All rights reserved in respect of Circulation Journal, and in respect of EuroIntervention.

Received October 17, 2017; revised manuscript received January 8, 2018; accepted January 16, 2018; released online March 15, 2018 Time for primary review: 11 days

Department of Cardiology, Bern University Hospital, Bern (K.C.K., L.R., M.V., S.W.), Switzerland; Toho University, Ohashi Medical Center, Tokyo (M.N.), Japan; Deutsches Herzzentrum München, Technische Universität München, Munich (R.C., R.A.B.), Germany; Kurashiki Central Hospital, Kurashiki (K.K.), Japan; Ferrarotto Hospital, University of Catania, Catania (D.C.), Italy; The Lambe Institute for Translational Medicine and Curam Saolta University Healthcare Group, Galway (W.W.), Ireland; Wakayama Medical University, Wakayama (T.A.), Japan; Interventional Cardiology Unit, Azienda Ospedaliera Papa Giovanni XXIII, Bergamo (G.G.), Italy; and DZHK (German Centre for Cardiovascular Research), partner site Munich Heart Alliance, Munich (R.A.B.), Germany

Mailing address: Konstantinos C. Koskinas, MD, MSc, FESC, Department of Cardiology, Bern University Hospital, 3010 Bern, Switzerland. E-mail: Konstantinos.koskinas@insel.ch

ISSN-1346-9843 All rights are reserved to the Japanese Circulation Society. For permissions, please e-mail: cj@j-circ.or.jp 
high-risk characteristics defined by IVUS, ${ }^{6,7}$ near-infrared spectroscopy (NIRS), ${ }^{8}$ and OCT $^{9}$ have been associated with subsequent adverse clinical outcomes, and ongoing studies are evaluating whether detection of high-risk plaques with these modalities may be clinically useful.

The perceptions of the global interventional community concerning the adoption of IVI in current practice are poorly defined. Moreover, although there is some evidence of geographic variations in the clinical use of IVI in patients undergoing PCI, ${ }^{10}$ this issue has not been assessed systematically. In addition, against a background of controversy regarding the usefulness of IVI for plaque assessment, ${ }^{11}$ little is known about the opinion of practicing interventional cardiologists in this respect. Against this background, the European Association of Percutaneous Coronary Interventions (EAPCI) and Japanese Association of Cardiovascular Interventions and Therapeutics (CVIT) undertook a webbased clinical practice survey with the aim of evaluating the views of the cardiology community on the clinical use of coronary IVI.

\section{Methods}

A voluntary survey was jointly performed by the EAPCI and CVIT. The survey was conducted using a free webbased tool (SurveyMonkey, Palo Alto, CA, USA) and comprised multiple-choice questions. The questionnaire (Tables S1,S2) was drafted by the EAPCI Scientific Documents Committee and approved by the EAPCI and CVIT boards. It was not mandatory to respond to all questions. The survey could be filled out anonymously, but respondents had the opportunity to provide their email address and receive a summary of the information collected. Invitations were sent on 5 June 2017 to 25,776 EAPCI and 6,117 CVIT email recipients. Reminders were issued on 12 and 29 June, and the survey closed on 8 July 2017.

Results are reported as numbers (percentages) and were compared using Chi-squared tests. Results were stratified for subgroups of interest including region or country of practice and level of interventional experience. Two-sided $\mathrm{P}<0.05$ was considered statistically significant. Analyses were performed using SPSS version 17.0 (SPSS Inc., Chicago, IL, USA).

\section{Results}

\section{Respondent Characteristics}

Of the 31,893 invitations sent, survey responses were received from 1,105 individuals $(3.5 \%)$. Personal and professional information with regard to age, geographic region of practice, and professional status was provided by 1,078 individuals $(97.6 \%)$. Respondent characteristics are summarized in the Table. Participants were more frequently practicing at university hospitals $(42.0 \%) ; 36.6 \%$ were working at low-volume centers $(<400$ PCIs performed per year), $39.2 \%$ were working in intermediate-volume centers (between 400 and 1,000 PCIs per year), and 24.2\% were working in high-volume centers (>1,000 PCIs per year). Respondents were predominantly interventional cardiologists with $>10$ years experience $(68.7 \%)$.

As shown in Figure S1, 34.7\% of respondents were from Europe and $52.0 \%$ were from Asia. Countries with greatest contribution were Japan $(45.4 \%)$, Italy $(6.3 \%)$, the US $(4.0 \%)$, the UK $(3.3 \%)$, Germany $(3.0 \%)$, and Switzerland $(2.1 \%)$. For region-related stratified analyses, respondents

\begin{tabular}{|lc|}
\hline Table. Respondent Characteristics & \\
Age group (years) (1,099 responses) & \\
$\quad<40$ & $246(22.4)$ \\
$40-50$ & $450(40.9)$ \\
$\quad>50$ & $403(36.7)$ \\
Professional status (1,097 responses) & \\
$\quad$ Interventional cardiologist with >10 years & $754(68.7)$ \\
$\quad$ experience & \\
Interventional cardiologist with 5-10 years & $181(16.5)$ \\
experience & \\
Interventional cardiologist with <5 years & $75(6.8)$ \\
experience & \\
$\quad$ Non-interventional cardiologist & $27(2.5)$ \\
Cardiologist in training & $21(1.9)$ \\
Other & $39(3.6)$ \\
Region (1,088 responses) & \\
Europe & $377(34.7)$ \\
Asia & $566(52.0)$ \\
Africa & $18(1.6)$ \\
North America & $64(5.9)$ \\
South America & $44(4.0)$ \\
Australia & $19(1.8)$ \\
Institution (1,091 responses) & \\
University hospital & \\
Non-academic public hospital & \\
Private institution & $456(41.8)$ \\
\hline Data are given as $n$ (\%). & $348(31.9)$ \\
& $287(26.3)$ \\
\hline
\end{tabular}

were grouped as European $(\mathrm{n}=377 ; 34.7 \%)$, Japanese $(\mathrm{n}=489 ; 45.4 \%)$, or participants from other regions $(\mathrm{n}=215 ; 19.9 \%)$.

\section{IVI for Guiding Interventions}

Overall, $96.1 \%$ of respondents had personal experience with intracoronary imaging $(95.5 \%$ with IVUS, $69.8 \%$ with OCT, and $7.9 \%$ with NIRS). There were no substantive region-related differences concerning personal experience with each modality.

Most respondents (51.3\%) reported using coronary IVI in $>15 \%$ of cases (Figure 1A). There were significant differences in usage according to the country of practice (Figure 1B): IVI was used more frequently in clinical practice ( $>15 \%$ of caseload guided by IVI) in Japan compared with Europe or other regions ( $96.6 \%$ vs. $10.4 \%$ and $21.0 \%$, respectively; $\mathrm{P}<0.001)$. There were also significant differences in usage according to the interventional experience of respondents (Figure 1C): IVI was used more frequently in clinical practice ( $>15 \%$ of caseload guided by IVI) by operators with longer interventional experience $(>10$ years: $58.9 \%$; $5-10$ years: $48.9 \%$; $<5$ years: $16.9 \%$; $\mathrm{P}<0.001$ ).

The most frequently reported indications for IVI were optimization of the procedural result of stenting in selected cases (i.e., post-procedural imaging; 88.5\%), guidance of clinical decision making and procedural strategy planning in selected cases (preprocedural imaging; 79.6\%), and guidance of left main interventions $(77.0 \%)$. The least common reported indication was assessment of the severity of intermediate non-left main lesions (27.1\%; Figure 1D). Most respondents reported perceived equipoise regarding the choice between IVUS and OCT for guidance of coronary intervention; $56.5 \%$ of respondents believed that the value 

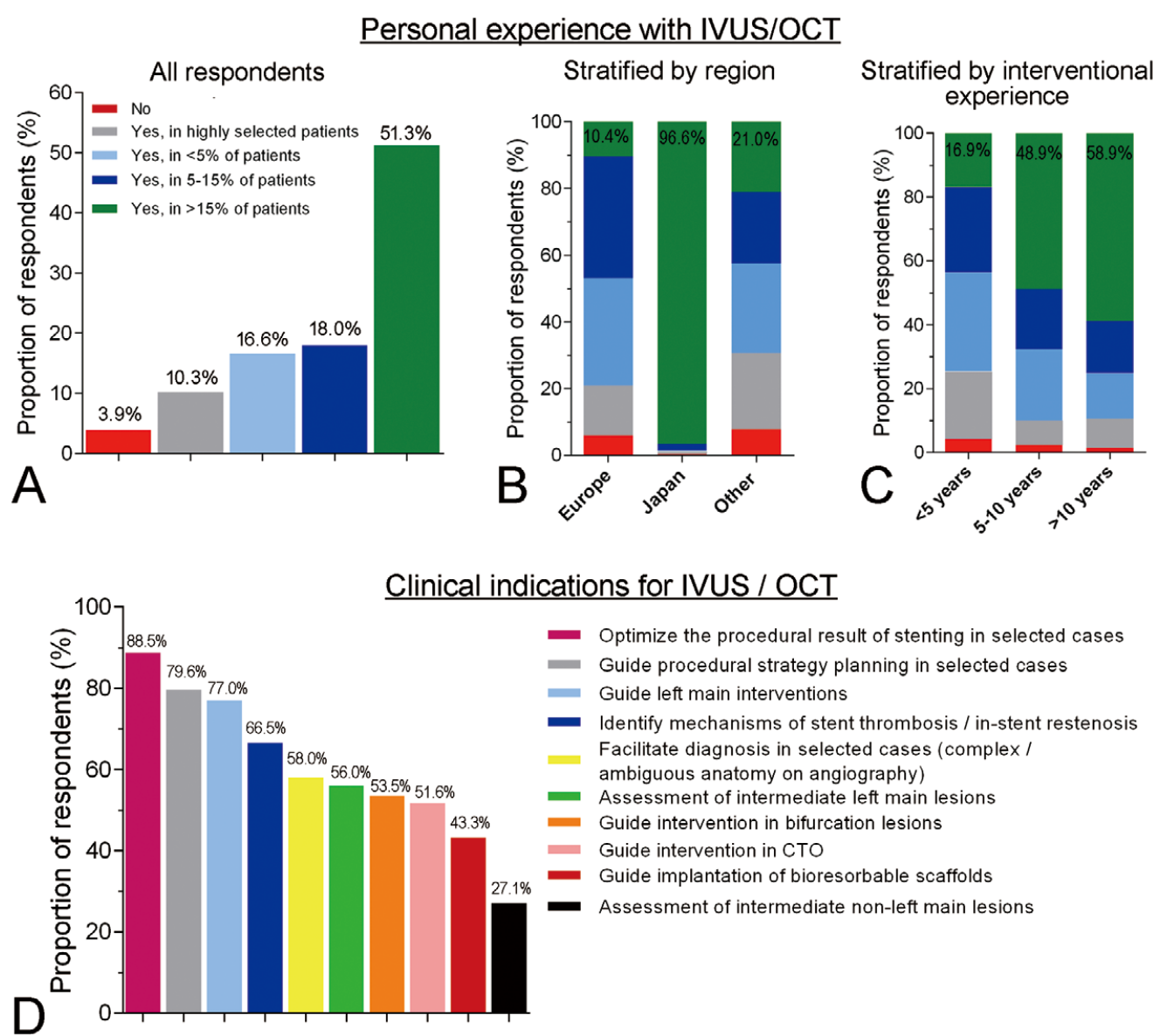

Figure 1. (A-C) Personal experience with intravascular ultrasound (IVUS) and/or optical coherence tomography (OCT). Respondent answers are shown to the question, "Do you have personal experience with performing IVI (IVUS, OCT, NIRS)? (Only 1 answer possible.)" in the overall sample (A), as well as stratified by region (B) and years of interventional experience (C). IVI, intravascular imaging; NIRS, near-infrared spectroscopy. (D) Clinical indications for IVUS/OCT. Respondents were asked, "In your opinion, what are the clinical indications for IVUS or OCT? (More than 1 answer possible.)". CTO, chronic total occlusion.

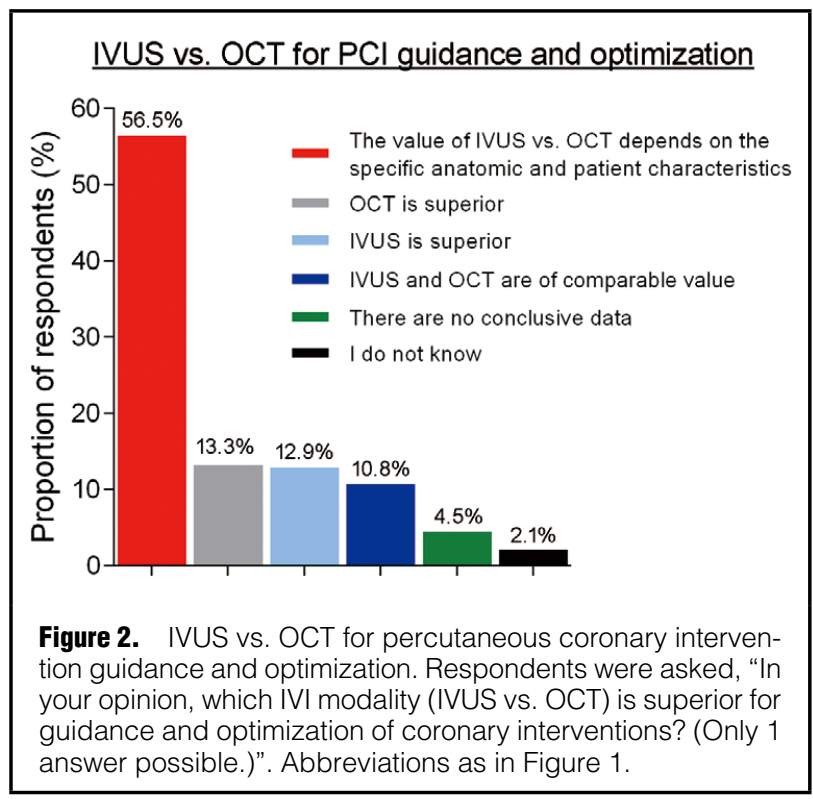

of IVUS vs. OCT depends on the specific anatomic setting and patient characteristics, whereas few considered either OCT $(13.3 \%)$ or IVUS $(12.9 \%)$ to be superior for PCI guidance and optimization (Figure 2). Regarding the assessment of intermediate left main lesions, IVUS was considered superior by $31.8 \%$ of respondents, whereas $28.4 \%$ of participants considered a non-imaging approach based on fractional flow reserve (FFR) superior; $24.8 \%$ of respondents considered these 2 modalities of comparable value in decision making and $15.0 \%$ responded that there is no conclusive evidence from comparative analyses.

Available evidence regarding IVI for PCI guidance and optimization was judged as good, average, and poor by $36.3 \%, 45.7 \%$, and $12.9 \%$ of operators, respectively, without significant region-related differences. The vast majority $(91.3 \%)$ responded that IVI has the potential to improve clinical outcomes of PCI (with similar proportions across regions), although $42.6 \%$ believed that current evidence in this respect is not definitive (Figure 3A). The proportion of respondents who were not convinced about the potential of catheter-based imaging to improve clinical outcomes was lower for respondents from Japan (5.3\%) compared 


\section{Imaging-guided $\mathrm{PCl}$ and clinical outcomes}
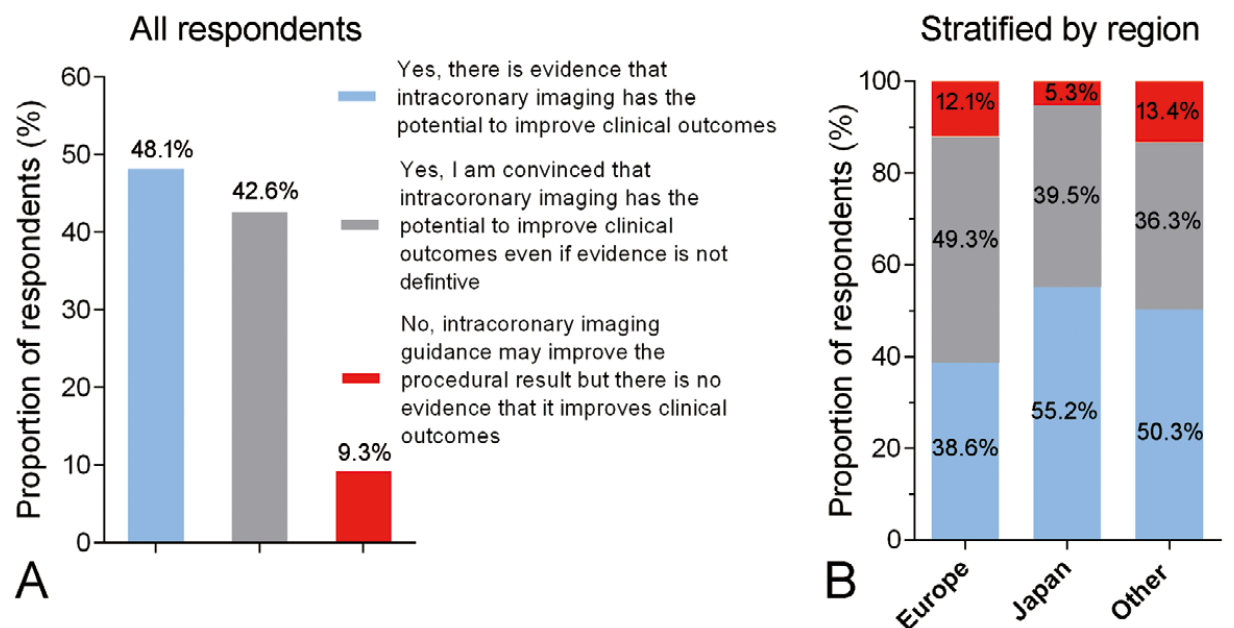

Figure 3. Imaging-guided percutaneous coronary intervention and clinical outcomes. Respondent answers are shown to the question, "Do you believe that IVUS or OCT guidance for coronary interventions improves clinical outcomes compared with angiography-only guidance? (Only 1 answer possible.)" for $(\mathbf{A})$ the overall sample and (B) stratified by region. Abbreviations as in Figure 1.

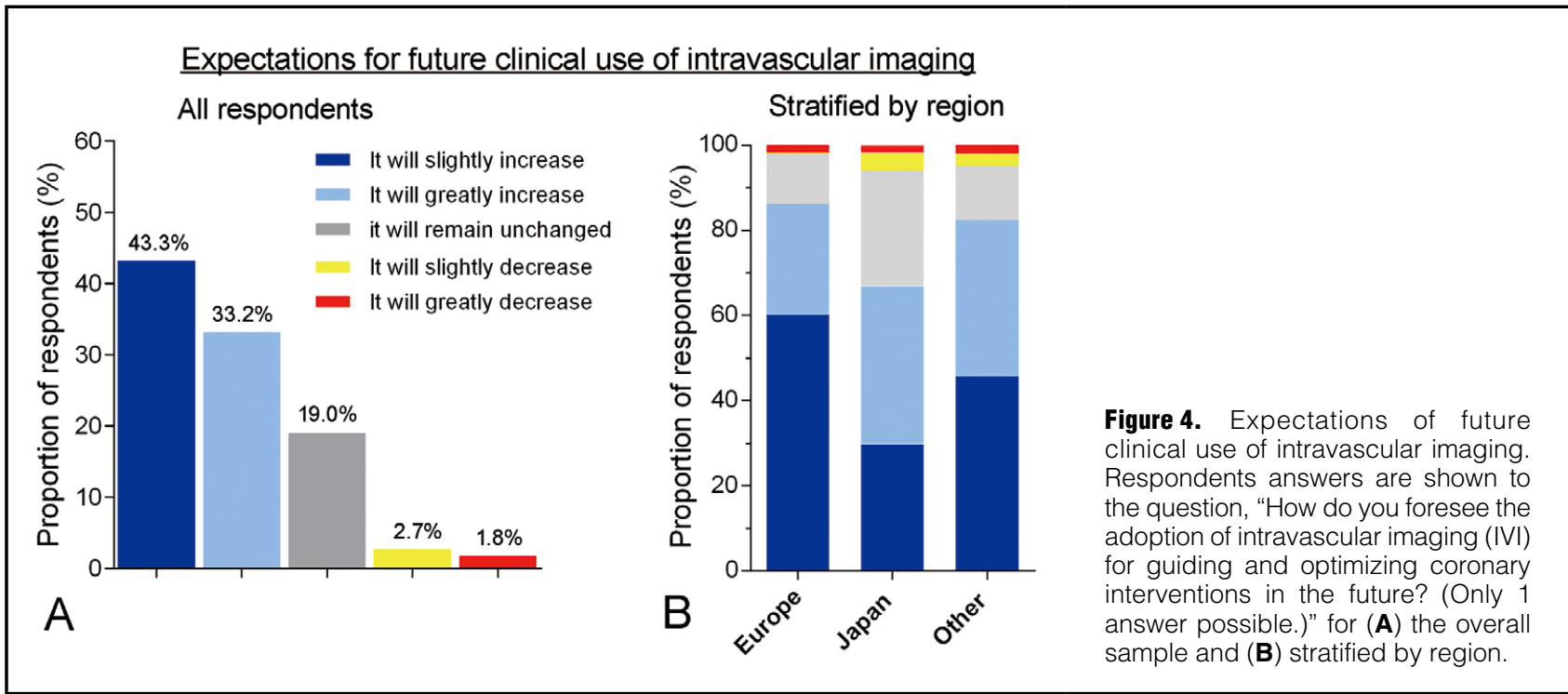

with Europe $(12.1 \%)$ or other regions $(13.4 \%$; $\mathrm{P}<0.01$; Figure 3B). Most respondents $(73.5 \%)$ estimated that the use of IVI will increase in the future, either slightly $(43.3 \%)$ or greatly $(33.2 \%$; Figure 4). Regarding research in the field, $35.3 \%$ responded that future studies should determine specific criteria for corrective measures in case of abnormal imaging findings (e.g., to define thresholds of malapposition or underexpansion that justify post-dilatation, or the extent of edge dissection to justify implantation of a new stent), and $22.9 \%$ believed that studies should focus on head-to-head comparison of IVUS vs. OCT guidance in specific patient and lesion subsets (Figure 5).

High cost was the most commonly reported reason limiting the clinical use of IVI (65.9\%), although this was less common among respondents from Japan (57.1\%) vs. Europe $(77.9 \%)$ or other regions $(84.0 \% ; \mathrm{P}<0.01)$. Other common reasons included prolongation of the procedure $(35.0 \% ; 22.6 \%$ of Japanese vs. $52.6 \%$ of European respondents; $\mathrm{P}<0.001)$ and reimbursement issues $(29.3 \%$; without significant region-related differences). Respondents from Japan, compared with Europe or other regions, less frequently cited lack of training, absence of established criteria for corrective measures, and absence of established clinical value of IVI as limiting factors (Figure 6). Risk of complications and absence of established clinical value were overall the least commonly reported limiting factors (9.5\% and $8.3 \%$, respectively; Figure 6). Respondents with $<5$ years interventional experience more commonly reported 


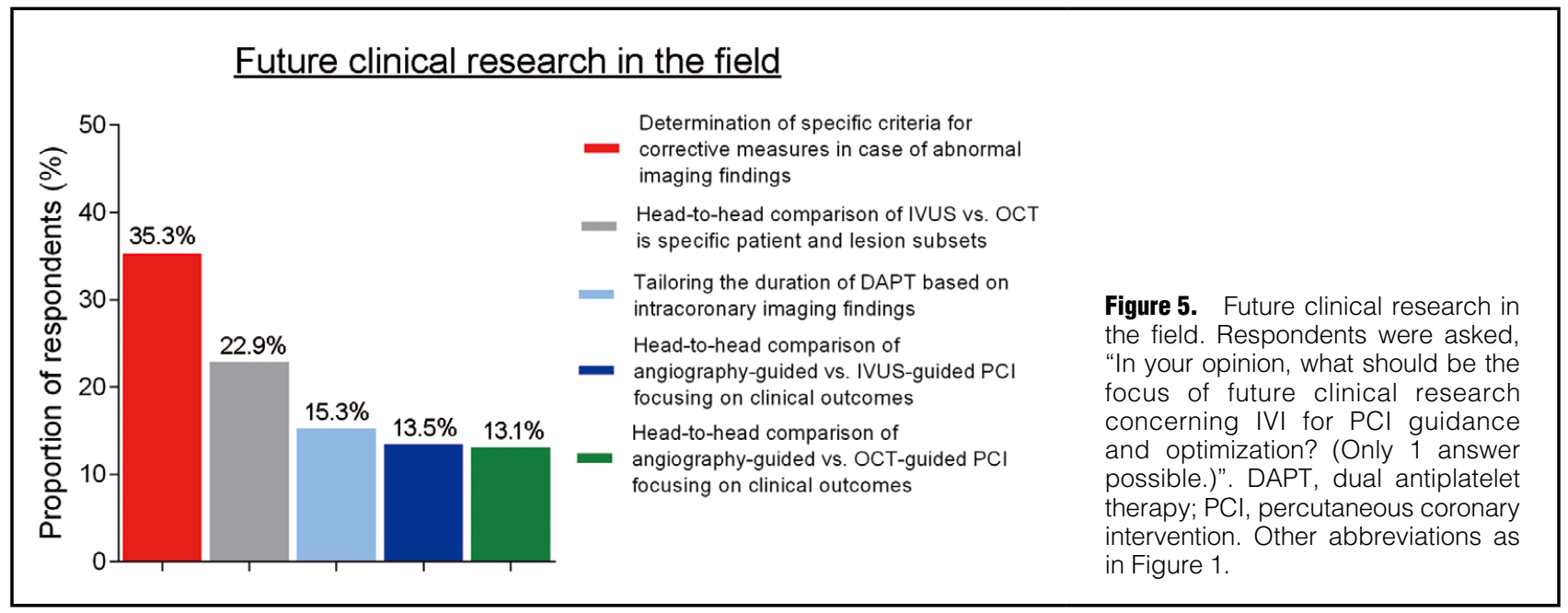

prolongation of the procedure $(56.9 \%$ vs. $35 \%$ in the overall sample) and lack of training in use and interpretation of these modalities $(30.8 \%$ vs. $17.1 \%$ overall) as limiting factors, without differences regarding the perceived risk of procedural complications $(10.8 \%$ vs. $9.5 \%$ of all respondents).

\section{IVI of Native Atherosclerosis}

Current evidence regarding coronary IVI for detection of vulnerable plaques was rated as good, average, and poor by $27.3 \%, 43.9 \%$, and y $28.8 \%$ of respondents, respectively (Figure 7A,B). A multimodality approach was considered more suited to detect vulnerable plaques by $38.1 \%$ of respondents, followed by OCT $(33.8 \%)$, IVUS-based technologies $(10.3 \%)$, and NIRS (7.1\%). When appraising the current and future role of IVI for evaluation of native coronary atherosclerosis, $60.6 \%$ responded that it may become an important tool to detect and potentially treat high-risk plaques (no significant differences across regions), and $46.3 \%$ responded that serial imaging is clinically useful to evaluate the effects of medical interventions on plaque progression or regression and changes in plaque morphology (60.7\% of respondents from Japan vs. 31.5\% from Europe and $38.0 \%$ from elsewhere; $\mathrm{P}<0.001$ ). For $21.5 \%$ of respondents, IVI of native atherosclerosis is seen purely as a research tool that will not become useful to detect or potentially treat high-risk plaques $(15.1 \%$ vs. $27.6 \%$ and $24.5 \%$ of respondents from Japan vs. Europe and other regions, respectively; $\mathrm{P}<0.01$; Figure $7 \mathrm{C}, \mathrm{D})$.

Sensitivity analyses in Asian respondents comparing Japanese vs. non-Japanese participants in relation to personal experience with performing IVI, perceptions regarding the effect of IVUS or OCT guidance on clinical outcomes, and evaluation of current evidence regarding IVI for detection of vulnerable plaques are shown in Figures S2-S4.

\section{Discussion}

Observational studies and randomized trials have provided compelling evidence of the value of coronary IVI for improving PCI outcomes in properly selected patients. The present clinical practice survey evaluated current adoption rates, operator perceptions, and expectations concerning IVI in interventional practice. In a selected sample of predominantly experienced interventional cardiologists, we found a high level of confidence in the clinical value of IVUS and OCT for guiding and optimizing coronary interventions, and reasonable consistency between operatorreported practice patterns and current guideline recommendations. The present study also highlights perceived unmet needs concerning research in the field and documents substantial geographic variability in practice patterns, findings that deserve consideration in future study planning and upcoming recommendations by international societies. Finally, this survey shows a relatively favorable predisposition regarding the potential of these modalities to detect vulnerable plaques, although critical responses in this respect were not uncommon.

Although angiography has been the long-time workhorse for coronary interventions, it provides only 2-dimensional representation of the coronary anatomy without direct information on vessel dimensions, burden of atherosclerosis, or stent-related mechanical problems. In the present survey, reported indications for adjunctive catheter-based imaging largely match current recommendations on the clinical use of IVUS and OCT, ${ }^{5}$ suggesting awareness of the strengths, limitations, and recommended use of these modalities in interventional practice. Several factors perceived to limit the use of IVI were identified, including primarily high cost, reimbursement issues and prolongation of the procedure. These considerations should be interpreted in light of differences in reimbursement policies across countries (e.g., more liberal reimbursement in Japan). However, one should also consider that: (1) the clinical use of IVUS was reportedly low, even in higher-income countries without restrictive limitations in reimbursement (e.g., IVI was used frequently by $9.1 \%$ of respondents in Switzerland vs. $10.4 \%$ of respondents from Europe overall); and (2) IVUS-guided PCI appears to be a cost-effective approach according to a dedicated economic analysis. ${ }^{12}$ Therefore, claims of high cost may not fully explain the relatively limited use of IVI in countries outside Japan. Clinical use of these invasive modalities was overall considered safe, and the risk of procedural complications was infrequently reported as a limiting factor, even among less-experienced interventional cardiologists. Notably, lack of training in use and interpretation of these techniques and prolongation of the procedure were more common concerns for operators with 


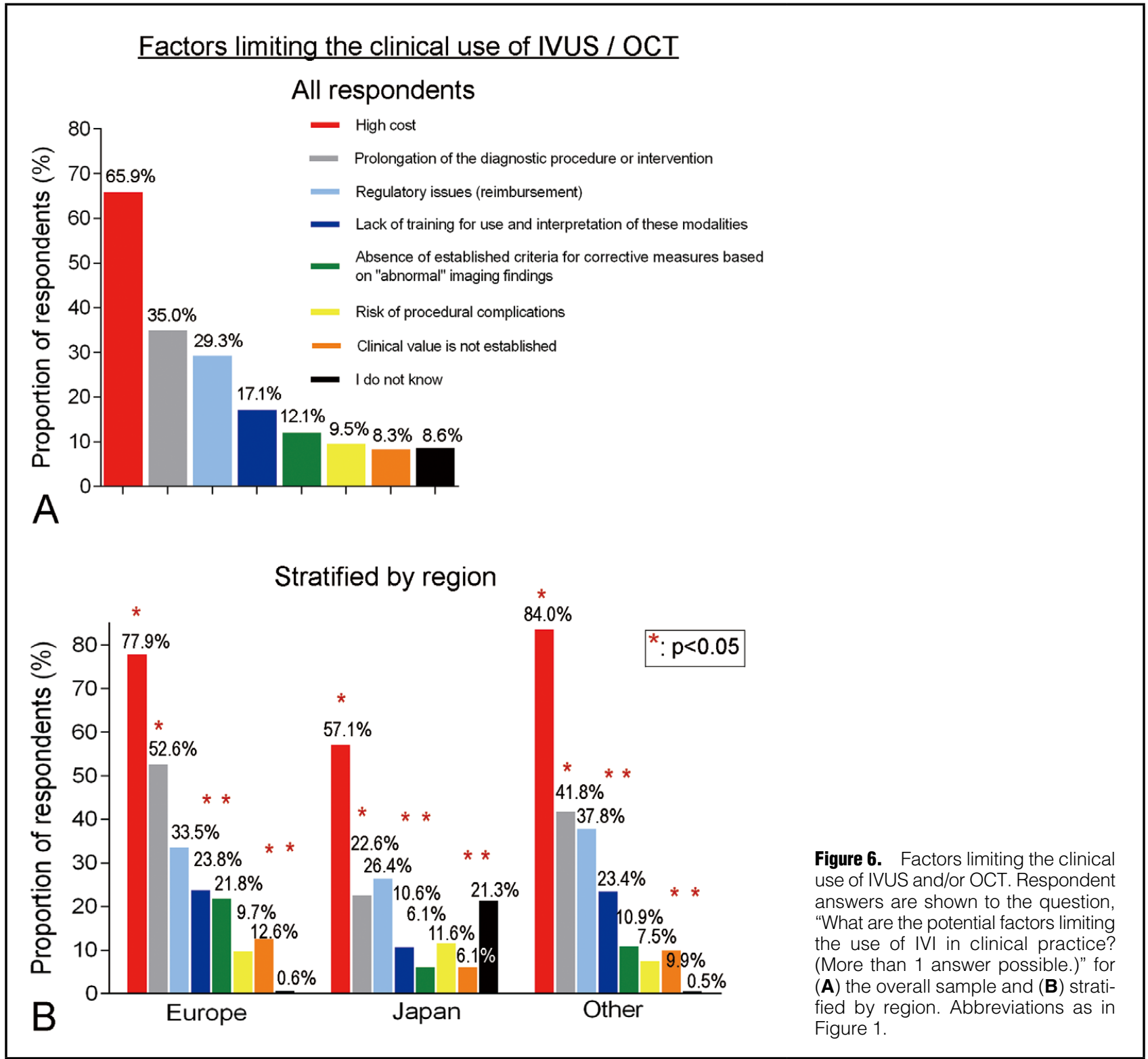

fewer years of experience, which likely accounts, in part, for the less frequent use of IVI in those with less experience compared with more-experienced operators.

IVUS and OCT each have inherent strengths and limitations (e.g., higher resolution at the expense of lower penetration for OCT). These modalities appear to perform comparably with regard to their effect on the procedural result, ${ }^{13}$ as well as on mid-term clinical outcomes of coronary interventions. ${ }^{\mathbf{1 4}}$ Therefore, selective utilization of IVUS or OCT in appropriate patient and lesion subsets during PCI procedures has been advocated as a reasonable approach. ${ }^{15}$ Along these lines, most respondents in the present survey concurred that the value of IVUS vs. OCT depends on the specific anatomic setting and patient characteristics; hence, selection of the modality should be individualized accordingly. Importantly, these responses were derived from a population with high rates of personal experience with IVI $(>95 \%)$ and in particular with both IVUS and OCT (almost $70 \%$ of operators).
A total of 9 out of 10 respondents were convinced about the potential of IVI to improve clinical outcomes beyond improving the acute procedural result. This is in accordance with meta-analyses of randomized trials showing improved clinical outcomes (driven by a reduction of ischemia-driven target lesion revascularization) with IVUS- vs. angiographyguided PCI with drug-eluting stents. ${ }^{16,17}$ Three-quarters of respondents had a positive outlook and expected further expansion in the use of these modalities in the future. The need to determine specific criteria for corrective measures in response to "abnormal" imaging findings was identified as the main unmet need in research in the field. Given the relative paucity of data in this respect, dedicated studies should determine specific cut-offs to differentiate clinically relevant abnormalities that warrant additional intervention from those that may be left untreated with a low risk of adverse clinical sequelae (e.g., the extent of malapposition or stent underexpansion where post-dilatation is indicated).

IVUS and OCT are known to be used widely in Japan. ${ }^{10}$ 


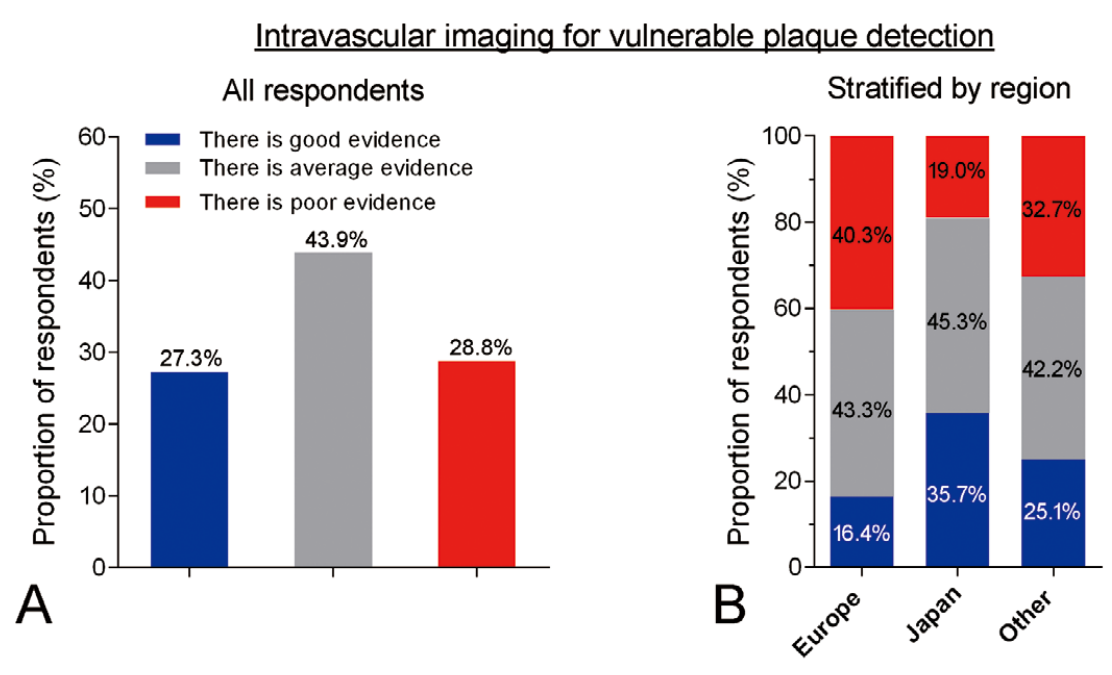

Current and future role of intravascular imaging of native atherosclerosis
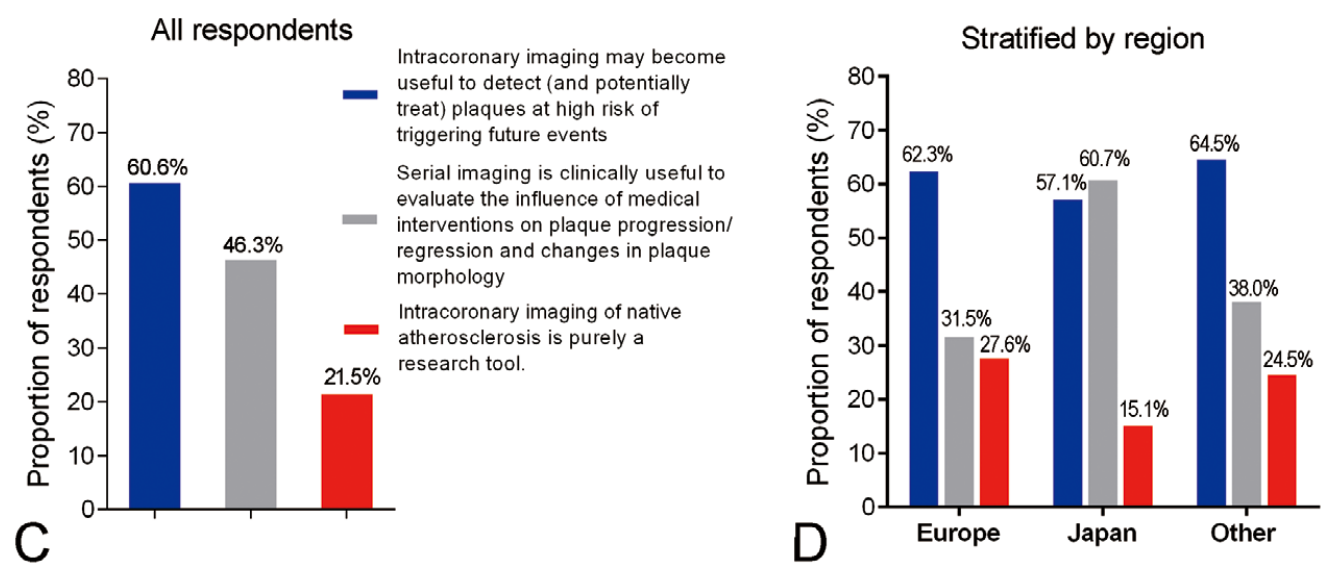

Figure 7. (A,B) Respondents' views regarding intravascular imaging (IVI) for vulnerable plaque detection. Recent studies (the PROSPECT study) and ongoing investigations (PROSPECT II, Lipid Rich Plaque) have focused on plaque characteristics of lesions associated with subsequent clinical events. In the present study, respondents were asked, "How do you judge current evidence regarding IVI for detection of "vulnerable" plaques, i.e., plaques perceived to be at high risk of triggering future cardiovascular events? (Only 1 answer possible.)". Responses are shown in the overall sample (A) and stratified by region (B). (C,D) Respondents' views of current and future roles of IVI for native atherosclerosis. IVI allows quantification of plaque burden and assessment of plaque morphology of native atherosclerotic lesions. Respondents were asked, "How do you consider the current and future role of IVI (IVUS, OCT, NIRS) for evaluation of native coronary atherosclerosis? (More than 1 answer possible.)". Responses are shown in the overall sample $(\mathbf{C})$ and stratified by region (D).

The present survey provides direct comparisons among operators from different regions around the world and highlights possible factors underlying these geographic variations. Most strikingly, almost all Japanese operators $(97 \%)$ replied that they use IVUS or OCT in a substantial proportion $(>15 \%)$ of patients they treat, compared with 1 in 10 European respondents. Against this background, the fact that many Japanese respondents predicted a further increase in the use of IVI is a striking finding that likely reflects the markedly favorable predisposition and high expectations from IVI modalities in Japan. Although participants of the present survey were overall favorably predisposed concerning the clinical benefit afforded by these modalities, this was even more pronounced among operators from Japan and could thereby explain, in part, the marked heterogeneity in adoption patterns. Along the same lines, the proportion of respondents from Japan who considered high cost a relevant limitation was lower compared with other countries. Collectively, although the findings of differing perceptions regarding the cost and clinical value of IVUS and OCT or training in these modalities provide some insights into the observed geographic heterogeneity, they cannot fully explain the substantial variation in the clinical use of IVI in Japan vs. other countries.

In contrast with IVUS or OCT in the context of PCI procedures, catheter-based imaging of atherosclerotic lesions that do not qualify for immediate treatment has not entered clinical practice. So-called high-risk or vulnerable plaque characteristics as assessed by means of IVUS-virtual histology or NIRS have been associated with subsequent clinical outcomes with high negative, but low positive predictive value. ${ }^{68}$ The concept of assessing high-risk 
plaques and the potential clinical utility of this approach have remained controversial. ${ }^{11}$ In the present survey, almost half of respondents felt that there is average evidence to support invasive imaging for detection of vulnerable plaques, and considered multimodality imaging most appropriate for this purpose. ${ }^{17}$ Moreover, 6 of 10 responded that these techniques may become suitable to detect and potentially treat such lesions in the future, although the proportion of those who consider this approach purely a research tool is not negligible (20\%). Region-related differences were also observed in this respect; notably, an almost 2-fold lower proportion of respondents from Japan compared with elsewhere had a critical opinion and a 2-fold higher proportion from Japan saw potential clinical value of serial invasive imaging to assess temporal changes in plaque burden and composition.

\section{Study Limitations}

This study has several limitations. First, the generalizability of the present findings is limited by the small percentage of invited practitioners $(3.5 \%)$ who participated in the survey, such that the results are not necessarily representative of the opinion of the cardiology community. However, the proportionally low participation is consistent with previous EAPCI ${ }^{18}$ and other international surveys ${ }^{19}$ within the interventional community and is common, particularly in surveys targeting professionals at an advanced career stage. Second, we cannot exclude selection bias towards respondents positively predisposed to the use of IVI, because physicians with greater interest and personal involvement in these modalities may be more likely to participate in the survey. Third, the survey provides a snapshot of practice patterns and operator perceptions and cannot reflect actual use or address changes over time. Fourth, we cannot exclude the possibility that perceptions regarding the use of IVI for bioresorbable scaffold implantation may have changed following the withdrawal of the ABSORB scaffold (after the present survey had been completed). We recorded institutional PCI volume but not individual operator volume. The number of cardiologists across countries was not recorded. We did not capture data on IVI in specific settings (e.g., imaging for atherectomy devices). Finally, although participants from a large number of countries worldwide were included in the survey, the present findings are less representative for regions underrepresented in the survey sample.

\section{Conclusions}

In a selected sample of predominantly experienced interventional cardiologists participating in a web-based survey conducted by 2 professional associations, there was a high rate of personal experience with IVI in clinical practice. The most commonly identified indications for intracoronary imaging were optimization of stenting, procedural/strategy guidance and guidance of left main interventions. Variability in practice patterns is substantial according to geographic region and interventional experience of the operator. Further studies should explore reasons for this variability in more detail.

\section{Acknowledgments}

The authors express their gratitude to the staff of Europa Organisation/ EuroPCR for their valuable support during survey development.

\section{Conflict of Interest}

M.N. reports receiving honoraria from Terumo, Volcano, and Abbott. L.R. reports receiving research grants (awarded to the institution) from Abbott. R.C. reports receiving a fellowship stipendium from The Irish Board for Training in Cardiovascular Medicine supported by MSD. W.W. reports receiving grants from St Jude - Abbott, Terumo, grants and personal fees from MicroPort, and grants and personal fees from Biotronik, and is a cofounder of Argonauts and a past non-executive board member of Genae and Celyad. M.V. reports receiving grants from Astra Zeneca and personal fees from Abbott, Amgen, and Beyer. G.G. reports receiving grants and personal fees from St Jude and research grants from Boston Scientific. S.W. reports receiving grants from Biotronik, Boston Scientific, Bracco Pharmaceutical, Edwards Lifesciences, Medtronic, Terumo Inc., and St Jude Medical. R.A.B. reports receiving personal fees from B. Braun Melsungen and Biotronik, grants and personal fees from Boston Scientific, and grants from Heartflow. All other authors have no conflicts of interest to disclose relevant to the content of this manuscript.

\section{Funding}

No funding was obtained for the present study.

\section{References}

1. Koskinas KC, Ughi GJ, Windecker S, Tearney GJ, Räber L. Intracoronary imaging of coronary atherosclerosis: Validation for diagnosis, prognosis and treatment. Eur Heart $J$ 2016; 37: $524-535$.

2. Witzenbichler B, Maehara A, Weisz G, Neumann FJ, Rinaldi MJ, Metzger DC, et al. Relationship between intravascular ultrasound guidance and clinical outcomes after drug-eluting stents: The Assessment of Dual Antiplatelet Therapy With DrugEluting Stents (ADAPT-DES) study. Circulation 2014; 129: 463470 .

3. Meneveau N, Souteyrand G, Motreff P, Caussin C, Amabile N, Ohlmann P, et al. Optical coherence tomography to optimize results of percutaneous coronary intervention in patients with non-ST-elevation acute coronary syndrome: Results of the multicenter, randomized DOCTORS study (Does Optical Coherence Tomography Optimize Results of Stenting). Circulation 2016; 134: 906-917.

4. Hong SJ, Kim BK, Shin DH, Nam CM, Kim JS, Ko YG, et al. Effect of intravascular ultrasound-guided vs angiography-guided everolimus-eluting stent implantation: The IVUS-XPL randomized clinical trial. JAMA 2015; 314: 2155-2163.

5. Windecker S, Kolh P, Alfonso F, Collet JP, Cremer J, Falk V, et al. 2014 ESC/EACTS guidelines on myocardial revascularization: The Task Force on Myocardial Revascularization of the European Society of Cardiology (ESC) and the European Association for Cardio-Thoracic Surgery (EACTS). Eur Heart J 2014; 35: 2541 2619.

6. Stone GW, Maehara A, Lansky AJ, de Bruyne B, Cristea E, Mintz GS, et al. A prospective natural-history study of coronary atherosclerosis. $N$ Engl J Med 2011; 364: 226-235.

7. Cheng JM, Garcia-Garcia HM, de Boer SP, Kardys I, Heo JH, Akkerhuis KM, et al. In vivo detection of high-risk coronary plaques by radiofrequency intravascular ultrasound and cardiovascular outcome: Results of the ATHEROREMO-IVUS study. Eur Heart J 2014; 35: 639-647.

8. Schuurman AS, Vroegindewey M, Kardys I, Oemrawsingh RM, Cheng JM, de Boer S, et al. Near-infrared spectroscopy-derived lipid core burden index predicts adverse cardiovascular outcome in patients with coronary artery disease during long-term followup. Eur Heart J 2018; 39: 295-302.

9. Niccoli G, Montone RA, Di Vito L, Gramegna M, Refaat H, Scalone G, et al. Plaque rupture and intact fibrous cap assessed by optical coherence tomography portend different outcomes in patients with acute coronary syndrome. Eur Heart $J$ 2015; 36: $1377-1384$

10. Hibi K, Kimura K, Umemura S. Clinical utility and significance of intravascular ultrasound and optical coherence tomography in guiding percutaneous coronary interventions. Circ J 2015; 79: 24-33.

11. Libby P, Pasterkamp G. Requiem for the "vulnerable plaque". Eur Heart J 2015; 36: 2984-2987.

12. Alberti A, Giudice P, Gelera A, Stefanini L, Priest V, Simmonds $\mathrm{M}$, et al. Understanding the economic impact of intravascular 
ultrasound (IVUS). Eur J Health Econ 2016; 1: 185-193.

13. Ali ZA, Maehara A, Généreux P, Shlofmitz RA, Fabbiocchi F, Nazif TM, et al. Optical coherence tomography compared with intravascular ultrasound and with angiography to guide coronary stent implantation (ILUMIEN III: OPTIMIZE PCI): A randomised controlled trial. Lancet 2016; 388: 2618-2628.

14. Kubo T, Shinke T, Okamura T, Hibi K, Nakazawa G, Morino $\mathrm{Y}$, et al. Optical frequency domain imaging vs. intravascular ultrasound in percutaneous coronary intervention (OPINION trial): One-year angiographic and clinical results. Eur Heart $J$ 2017; 38: 3139-3147.

15. Waksman R, Kitabata H, Prati F, Albertucci M, Mintz GS Intravascular ultrasound versus optical coherence tomography guidance. J Am Coll Cardiol 2013; 62(Suppl): S32-S40.

16. Elgendy IY, Mahmoud AN, Elgendy AY, Bavry AA. Outcomes with intravascular ultrasound-guided stent implantation: A meta-analysis of randomized trials in the era of drug-eluting stents. Circ Cardiovasc Interv 2016; 9: e003700.

17. Buccheri S, Franchina G, Romano S, Puglisi S, Venuti G, D'Arrigo $\mathrm{P}$, et al. Clinical outcomes following intravascular imaging-guided versus coronary angiography-guided percutaneous coronary intervention with stent implantation: A systematic review and Bayesian network meta-analysis of 31 studies and 17,882 patients. JACC Cardiovasc Interv 2017; 10: 2488-2498.

18. Valgimigli M, Costa F, Byrne R, Haude M, Baumbach A, Windecker S. Dual antiplatelet therapy duration after coronary stenting in clinical practice: Results of an EAPCI survey.
EuroIntervention 2015; 11: 68-74.

19. Bertrand OF, Rao SV, Pancholy S, Jolly SS, Rodés-Cabau J, Larose E, et al. Transradial approach for coronary angiography and interventions: Results of the first international transradial practice survey. JACC Cardiovasc Interv 2010; 3: 1022-1031.

\section{Supplementary Files}

\section{Supplementary File 1}

Figure S1. Distribution of respondents in relation to region of practice.

Figure S2. Sensitivity analysis: personal experience with IVI among respondents from Asia.

Figure S3. Sensitivity analysis: perceived effect of IVI on PCI outcomes among respondents from Asia.

Figure S4. Sensitivity analysis: evaluation of current evidence regarding IVI for detection of vulnerable plaques among respondents from Asia.

Table S1. Survey questionnaire: general questions

Table S2. Survey questionnaire: specific questions

Please find supplementary file(s);

http://dx.doi.org/10.1253/circj.CJ-17-1144 